\title{
Advantages of a Double Three-Phase Winding Layout for a Dual Rotor E-Bike Motor Considering Third Current Harmonic Injection Technique
}

\author{
Daniele De Gaetano*, Dmitry Golovanov*, Giacomo Sala ${ }^{\dagger}$, Alessandro Galassini*, \\ Michele Degano* $^{* \ddagger}$, Hanafy Mahmoud ${ }^{\S}$, Chris Gerada*‡ \\ * Power Electronics, Machines and Control Group \\ University of Nottingham, Nottingham, UK \\ $\dagger$ Department of Electrical, Electronic, and Information Engineering \\ Univeristy of Bologna, Bologna, IT \\ $\ddagger$ Power Electronics, Machines and Control Group \\ University of Nottingham, Ningbo, Ningbo, CN \\ $\S$ Electric Power and Machines Department \\ Cairo University, Cairo, Egypt.
}

Email: daniele.degaetano2@nottingham.ac.uk

\begin{abstract}
The transportation electrification process is rapidly demanding for drivers with enhanced performance. Modern systems are moving from fossil fuel technologies toward hybrid and fully electric solutions. Consequently, in automotive and motorcycle sectors the complete electrification process requires significant power and energy capabilities, and high efficiency. Also, to minimize the mechanical power loss and save the battery energy, the weight and volume of the vehicle have to be minimized. These features are significantly stressed in race applications, where the power density of the drive and its efficiency has to push the limits of state of the art. In this work, a multiphase machine with dual rotor architecture is employed, aiming at maximizing the performance of a full electric motorcycle (E-Bike). In particular, it is already known that in multi-phase machines it is possible to inject high frequency current component to increase the power density of the drive. Therefore, the analytical model for the injection of a third harmonic in the currents waveforms is presented for a case study of E-Bike featuring a dual rotor with Halback permanent magnet array. The third current harmonic is injected keeping the same peak current, when compared with the control technique without injection. The three-phase and multiphase winding layout of the machine are compared with and without current injection control by means of analytical equations. Finally, finite element results are shown in order to validate the model, the advantage of the multiphase winding and the current injection technique.
\end{abstract}

\section{INTRODUCTION}

In transport applications the electrical machines torque capability is a very important aspect to be considered. This paper proposes a combination of a dual rotor, a multi-phase windings re-arrangement and the harmonic current injection technique. The target is the assessment of the torque capability for this project solution. It has been demonstrated that multi phase systems with current shift control can give significant benefits in terms of torque capability and ripple reduction [1].
In [2], the torque increment for a five-phase machine with unequal teeth and third current harmonic injection is studied. The authors in [3] compared two winding arrangements in two different machine geometries of three-phase dual rotor drives. In [4], an analysis of a nine-phase PM machine with different current waveforms and back electro-motive force (BEMF) combinations is carried out. In [5], the torque improvement was given by the fifth and seventh harmonic current injection. In [6], the third harmonic injection technique has been applied for a 9-phase machine and a rotor configuration with a highly non-sinusoidal BEMF. The proposed work aims to study how a dual three-phase systems and third current harmonic injection technique can influence the torque performance and efficiency of a dual rotor machine with fractional slots for E-Bike [7]. In sec. II, the dual three-phase configuration is presented while sec. III shows how the third harmonic current injection is applied for the proposed machine. In addition, the magnetomotive force harmonic spectrum genereted by currents is analysed for both three and dual three-phase configurations. Finally, in the sec. IV a comparison between three-phase and dual three-phase configurations with and without current harmonic injection is carried out showing pros and cons of the proposed solution.

\section{Preliminary AnAlyses}

\section{A. Dual rotor machine characteristics}

The dual rotor machine under investigation presents a rotor with Halbach configuration and a stator with Fractional Slots Concentrated Windings (FSCW). The main machine parameters are: 24 slots, 14 pole pairs, fundamental frequency of $2100 \mathrm{~Hz}$. The geometry is shown in Fig. 1. The multi phase winding configuration has been designed in order to allow a 
phase shift current control for improving the torque capability.

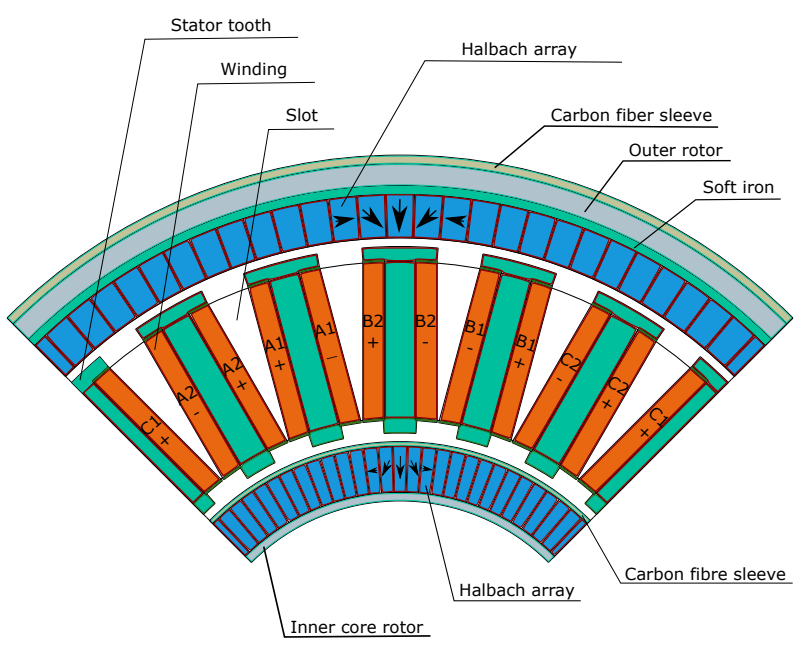

Fig. 1. Dual rotor machine sketch and winding configuration.

\section{B. BEMF at base angular speed}

In this work, the dual three-phase solution with a shift of 30 electrical degrees between the two different sets is adopted. This solution to increase the torque capability of the machine provided that the currents of both sets are controlled proprely. In Fig. 2, a BEMFs comparison, carried out by means of Finite Element Analysis (FEA), between three-phase and multi-phase configurations with linear material is given. The BEMFs for dual three-phase system is showing the 30 degrees shift between the same phase for the two different set of windings. In this motor, the Maximum Torque Per Ampere (MTPA) is achieved when the phase currents are aligned with the respective BEMFs because the d-axis reactance is equal to the q-axis one $\left(X_{d}=X_{q}\right)$. This corresponds to have both the three-phase subsystems operating with only their quadrature component of the currents. Thus, the currents have to be injected without shift with respect to the their own phase BEMF. Fig. 3 shows the current vector diagram for this configuration, in agreement with the theory. It is worth noticing that for dual three-phase configuration the BEMF amplitude is about half compared to three-phase one for the same number of turns per coil.

\section{3RD CURRENT HARMONIC INJECTION}

The third current harmonic generated by the inverter can be used to improve the average torque output. The proposed dual rotor with Halbach permanent magnet array presents a sinusoidal BEMFs so that the third harmonic component is not present as shown in Fig. 4. In [8] it is shown how to increase the torque performance by injecting the third harmonic without the third harmonic BEMF and working on the inverter only. The third current harmonic injection allows to reduce the current peak about $15 \%$, with respect to the fundamental only. Thus, it is possible to increase the current value, without exceeding the inverter current limit (Fig. 5). In this way, the peak current is constant but its RMS value is higher, therefore it leads to higher thermal loading. To have a more general understanding of the injection technique, the evaluation of the machine behaviour is carried out neglecting the non linear behaviour of the ferromagnetic materials.
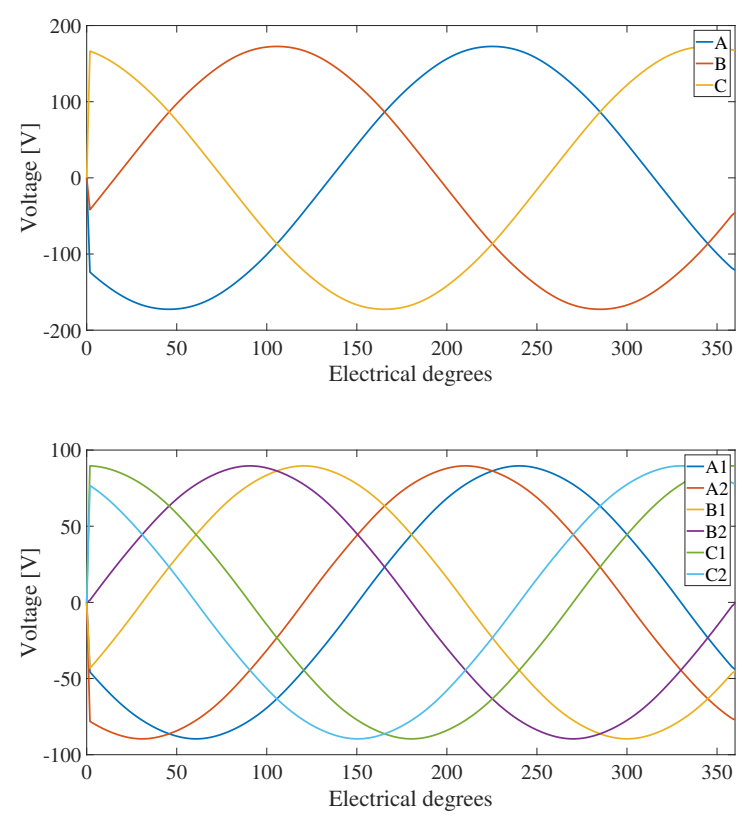

Fig. 2. BEMFs for 3-phase and 2x3-phase by FEA.

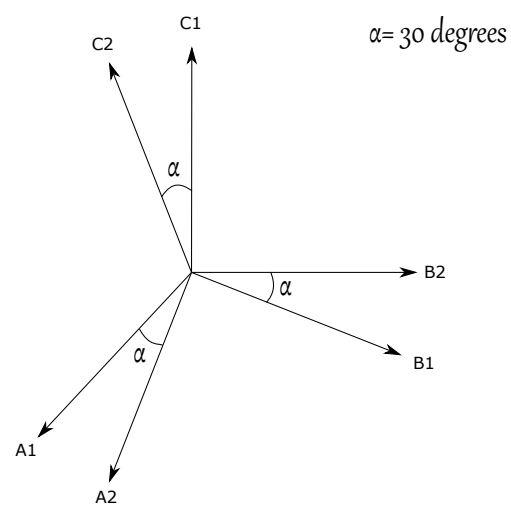

Fig. 3. Current vectors diagram for $2 \times 3$-phase system.

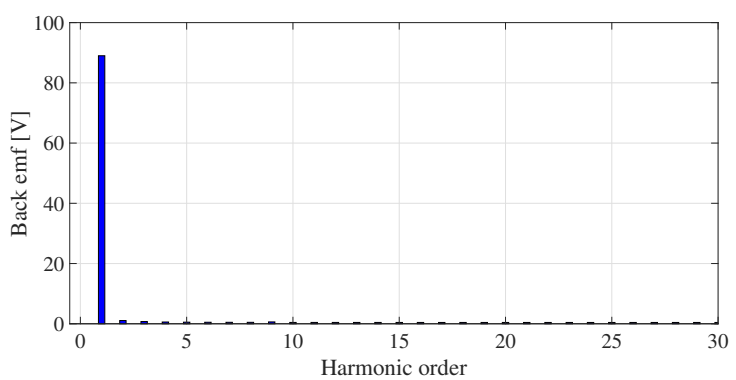

Fig. 4. FFT of one phase BEMF considering linear material. 


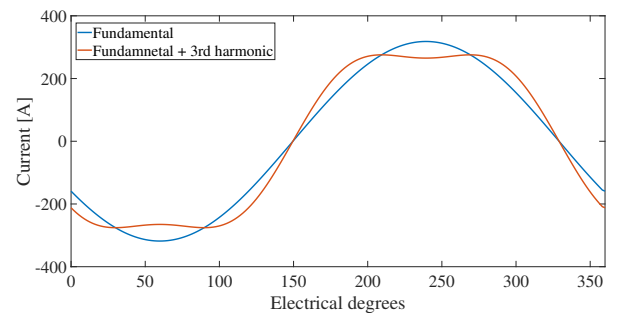

Fig. 5. Phase current waveforms with and without 3rd current harmonic injection.

\section{A. Third harmonic current injection at constant peak}

This subsection shows the main equations for the third harmonic injection for a dual three-phase machine. The equations are derived in [8]. The fundamental current feeding the machine is expressed as:

$$
i_{\theta}=I_{p} \sin (p \theta),
$$

and the current including the third harmonic injection is therefore:

$$
i_{\theta}=I_{\text {new }}\left[\sin (p \theta)+A_{3} \sin (3 p \theta)\right],
$$

where $I_{p}$ is the peak phase current, $I_{n e w}$ is the new peak phase current of the first harmonic component once the third harmonic is injected and $A_{3}$ is the amplitude of the third harmonic with respect to the fundamental one. The peak current can be calculated equating to zero the derivative of $i(\theta)$ with respect to $\theta$ :

$$
\frac{d i_{\theta}}{d \theta}=I_{p} p\left[\cos (p \theta)+3 A_{3} \cos (3 p \theta)\right]=0,
$$

The optimum current amplitude can be obtained in two conditions:

$$
\left\{\begin{array}{l}
\cos (p \theta)=0,0<A_{3} \leq \frac{1}{9} \\
\cos (p \theta)=\sqrt{\frac{9 A_{3}-1}{12 A_{3}}}, A_{3}>\frac{1}{9}
\end{array}\right.
$$

The phase current can be explicit:

$$
\left\{\begin{array}{l}
I_{p}=I_{\text {new }}\left(1-A_{3}\right), 0<A_{3} \leq \frac{1}{9} \\
I_{p}=I_{\text {new }} 8 A_{3}\left(\frac{1+3 A_{3}}{12 A_{3}}\right)^{1.5}, A_{3}>\frac{1}{9}
\end{array}\right.
$$

The new current $I_{\text {new }}(\theta)$ can be calculeted as:

$$
\left\{\begin{array}{l}
I_{\text {new }}=\frac{I_{p}}{1-A_{3}}, 0<A_{3} \leq \frac{1}{9} \\
I_{\text {new }}=\frac{1}{8 A_{3}}\left(\frac{1+3 A_{3}}{12 A_{3}}\right)^{-1.5} I_{p}, A_{3}>\frac{1}{9}
\end{array}\right.
$$

The torque for a general dual-three phase machine once the third current harmonic is injected can be written as:

$$
T=6 R l N\left[B_{1} k_{w 1} i_{n e w}+A_{3} B_{3} k_{w 3} i_{n e w}\right],
$$

with $m$ phases number, $R$ inner stator radius, $l$ active machine stack length, $N$ turns number per phase, $B_{1}$ and $B_{3}$ the flux density amplitude for the first and third harmonic, respectively. $k_{w 1}$ and $k_{w 3}$ are the winding factors for the first and third harmonic, respectively.
1) For the case $0<A_{3} \leq \frac{1}{9}$ the torque (7) can be re-arranged in the following way:

$$
T=6 R l N \frac{I_{p}}{1-A_{3}}\left[B_{1} k_{w 1}+A_{3} B_{3} k_{w 3}\right],
$$

the maximum torque value is determined when the third harmonic current amplitude, with respect to the fundamental one, is:

$$
A_{3}=\frac{1}{9}
$$

Therefore, the new current becomes:

$$
I_{\text {new }}=I_{p} \frac{9}{8} \text {. }
$$

2) For the case $A_{3}>\frac{1}{9}$ it is possible to get the best amplitude coefficient in order to increse the torque (7) applying Lagrange:

$$
\frac{d L\left(I_{\text {new }}, A_{3}\right)}{d I_{\text {new }}}=0 ; \frac{d L\left(I_{\text {new }}, A_{3}\right)}{d A_{3}}=0 .
$$

The new current and amplitude third harmonic coefficient with respect to the fundamental one for $A_{3}>\frac{1}{9}$ are:

$$
\begin{gathered}
A_{3}=\frac{1}{6-3 \frac{E_{3}}{E_{1}}} . \\
I_{\text {new }}=\frac{6-3 \frac{B_{3}}{B_{1}} \frac{k w_{3}}{k} w_{1}}{8}\left(\frac{3-\frac{B_{3}}{B_{1}} \frac{k w_{3}}{k w_{1}}}{4}\right)^{-1.5} I_{p} .
\end{gathered}
$$

where $E_{1}$ and $E_{3}$ are the first and the third harmonic BEMF, respectively. In the proposed case of study, the BEMFs ratio $\frac{E_{3}}{E_{1}}$ is almost zero. Therefore, the optimum amplitude of third harmonic component of current is $\frac{1}{6}$ with respect to the fundamental one. This amplitude is applied also for the threephase machine analyses.

\section{B. Magneto-motive force of the stator currents}

In this section, the magneto-motive force of the stator (MMFs) generated by a symmetrical multi phase system composed by $m$ phases which are fed by a symmetrical set of currents $\sum_{x=1}^{m} i_{x}=0$ and shifted with respect to each other by an angle of $\frac{2 \pi p}{m}$, with $\mathrm{p}$ the pole pairs number, is reported. The MMF can be calculated as in 14:

$$
M M F_{S}(\vec{i}, \lambda)=\vec{N}(\lambda)^{T} \vec{i},
$$

where $\vec{N}$ is a vector, which represent the winding functions for each phase and $\lambda$ is the spatial coordinate in a static reference frame. The winding function and current vectors are expressed in (15) and (16), respectively.

$$
\begin{gathered}
\vec{N}(\lambda)=\left[\begin{array}{c}
\vdots \\
\left.\sum_{h=0}^{\infty} N_{h} \cos \left(h p \lambda-\frac{2 \pi p h}{m} x-\lambda\right)\right] \\
\vdots
\end{array}\right] \\
\vec{i}(\lambda)=\left[\begin{array}{c}
\left.\sum_{\rho=0}^{\infty} I_{\rho} \cos \left(\rho \omega_{e l} t-\frac{2 \pi p h}{m} x-\psi\right)\right] \\
\vdots
\end{array}\right]
\end{gathered}
$$


TABLE I

MAGNETO-MOTIVE FORCE SPATIAL HARMONICS GENERATED BY DIFFERENT TEMPORAL CURRENT HARMONICS FOR A THREE-PHASE SYSTEM

\begin{tabular}{ccc}
\hline- & $\rho=1$ & $\rho=3$ \\
\hline$h=1$ & $\omega_{e l} t$ & 0 \\
$h=3$ & 0 & $3 \omega_{e l} t$ and $-3 \omega_{e l} t$ \\
$h=5$ & $-\omega_{e l} t$ & 0 \\
$h=7$ & $\omega_{e l} t$ & 0 \\
$h=9$ & 0 & $3 \omega_{e l} t$ and $-3 \omega_{e l} t$ \\
$h=11$ & $-\omega_{e l} t$ & 0 \\
$h=13$ & $\omega_{e l} t$ & 0 \\
$h=15$ & 0 & $3 \omega_{e l} t$ and $-3 \omega_{e l} t$ \\
$h=17$ & $-\omega_{e l} t$ & 0 \\
$h=19$ & $\omega_{e l} t$ & 0 \\
\hline
\end{tabular}

Substituting (15) and (16) in (14):

$$
\begin{aligned}
& M M F_{S}(\vec{i}, \lambda)=\sum_{x=1}^{m} \sum_{h=0}^{\infty} \sum_{\rho=0}^{\infty} N_{h} I_{\rho}\left\{\cos \left(n p \lambda-\frac{2 \pi p h}{m} x-\lambda\right)\right. \\
& \left.\cos \left(q \omega_{e l} t-\frac{2 \pi p h}{m} x-\lambda\right)\right\}
\end{aligned}
$$

The (17) can be rearranged in the following way:

$$
\begin{aligned}
& M M F_{S}(\vec{i}, \lambda)=\sum_{x=1}^{m} \sum_{h=0}^{\infty} \sum_{\rho=0}^{\infty} \frac{N_{h} I_{\rho}}{2}\{ \\
& \cos \left(n p \lambda+\omega_{e l} t \rho-\frac{2 \pi p h}{m} x-\frac{2 \pi p \rho}{m} x-\lambda-\psi\right)+ \\
& \left.\cos \left(n p \lambda+\omega_{e l} t \rho-\frac{2 \pi p h}{m} x+\frac{2 \pi p \rho}{m} x-\lambda+\psi\right)\right\}
\end{aligned}
$$

The MMFs can be written:

$$
\begin{aligned}
& M M F_{S}(\vec{i}, \lambda)=\sum_{h=0}^{\infty} \sum_{\rho=0}^{\infty} \frac{N_{h} I_{\rho}}{2} R e\{ \\
& e^{\left(n p \lambda+\omega_{e l} t \rho+\lambda_{h}-\psi\right)} \sum_{x=1}^{m} e^{-j \frac{2 \pi p(h+\rho)}{m} x}+ \\
& \left.e^{\left(n p \lambda-\omega_{e l} t \rho-\lambda_{h}+\psi\right)} \sum_{x=1}^{m} e^{-j \frac{2 \pi p(h-\rho)}{m} x}\right\}
\end{aligned}
$$

The stator generates a MMFs spectrum which presents superior order harmonics other than the fundamental one. Some harmonics are rotating in the same direction as the fundamental (direct component), whereas other harmonics are rotating in the opposite direction (inverse component). For each temporal harmonic $\rho$ there is a spectrum of spatial harmonics $h$. With this formulations, it is possible to calculate analytically which harmonic order will be present and its frequency for different winding configurations (three or multi phase) and with or without temporal harmonic current injection.

1) three-phase system: in table I are summarized the inverse and direct MMFs spatial harmonic components for different temporal harmonic current components for a classical three-phase system.
TABLE II

MAGNETO-MOTIVE FORCE SPATIAL HARMONICS GENERATED BY DIFFERENT TEMPORAL CURRENT HARMONICS FOR A DUAL THREE-PHASE SYSTEM

\begin{tabular}{ccc}
\hline- & $\rho=1$ & $\rho=3$ \\
\hline$h=1$ & $\omega_{e l} t$ & 0 \\
$h=3$ & 0 & $3 \omega_{e l} t$ \\
$h=5$ & 0 & 0 \\
$h=7$ & 0 & 0 \\
$h=9$ & 0 & $-3 \omega_{e l} t$ \\
$h=11$ & $-\omega_{e l} t$ & \\
$h=13$ & $\omega_{e l} t$ & 0 \\
$h=15$ & 0 & $3 \omega_{e l} t$ \\
$h=17$ & $-\omega_{e l} t$ & 0 \\
$h=19$ & $\omega_{e l} t$ & 0 \\
\hline
\end{tabular}

2) dual three-phase: using two three-phase subsystems with a shift between them of 30 electrical degrees, it is possible to get a six-phase arrangement. In table II are shown the main components of the MMFs for this configuration.

\section{Finite Element Analyses}

In this section, a comparison of the machine performance between three-phase and dual three-phase, with and without third current harmonic injection, is carried out by means of FE simulations. While sec. II is based on the assumption of linear material beahviour, the FE results are presented taking into account the non-linearities of the ferromagnetic materials (i.e. the iron saturation) in this section. The optimum amplitude of the third current harmonic for the proposed machine is $1 / 6$ of the fundamental because the third harmonic BEMF is not present (12). Therefore, the resulting phase currents with and without harmonic injection are displayed in Fig. 6 and Fig. 7, where also the common mode currents (CMC) of each threephase system are highlighted.

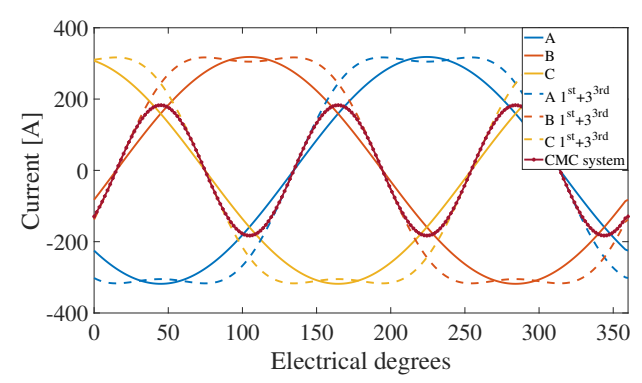

Fig. 6. Phase currents for the 3-phase arrangement.

\section{A. Analysis with only fundamental current component}

1) Torque: in Fig. 8 the torque for both three-phase and dual three-phase systems is shown. The average torque is higher for the multi-phase solution due to an improved winding factor. The dual three-phase system gives an important benefit also in terms of torque ripple which decreases from $4.5 \%$ to $0.84 \%$. Indeed, the dual three-phase arrangement 


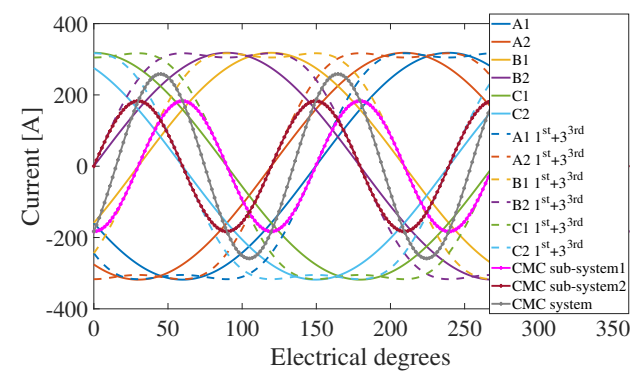

Fig. 7. Phase currents for the $2 \times 3$-phase arrangement.

does not generate the sixth harmonic torque component and the first harmonic component other than the fundamental is the twelfth according to the theory.
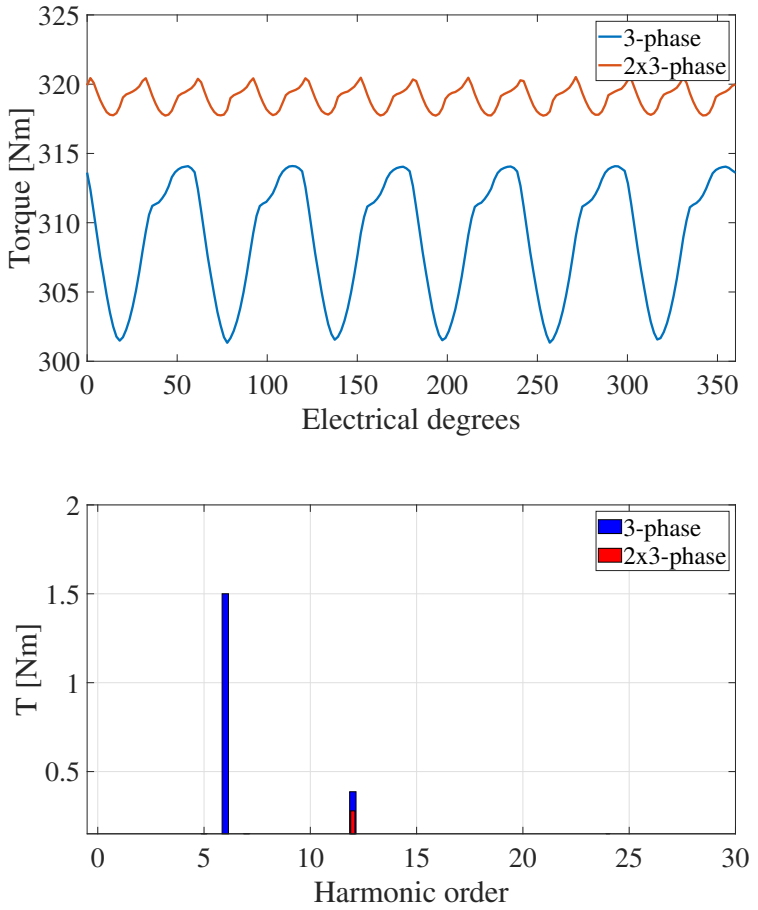

Fig. 8. Torque ripple waveforms for 3 -phase and $2 \times 3$-phase arrangement and corresponding FFT.

2) Efficiency: for the same configurations, the efficiency is calculated at the base angular speed $\omega$ with the following formula:

$$
\eta=\frac{\text { Poutput }}{\text { Poutput }+ \text { Losses }} 100=\frac{T \omega}{T \omega+\text { Losses }} 100,
$$

where $T$ is the average torque and $\omega$ is the angular base speed in $\mathrm{rad} / \mathrm{s}$. Table IV summarizes the losses and machine efficiency obtained from the FE results. The losses are similar for the two systems so that the efficiency results higher for dual three-phase configuration due to an higher output power

B. Analysis with third current temporal harmonic component

1) Torque: Fig. 9 shows the torque for both systems when the third current harmonic injection is applied. Both config- urations present an higher torque compared to the control technique without third current harmonic injection due to higher current in the machine. In this operating condition, the ripple is slightly higher compared to the solution without third current harmonic injection. Actually, the ripple increases of the $0.51 \%$ for the 3-phase arrangement whereas of the $0.30 \%$ for the $2 \times 3$-phase one. This phenomenon is due to the fact that the MMFs harmonic spectrum of the three-phase arragement presents more components than the dual three-phase one. This is highlighted even more in presence of third current harmonic injection (Table I and Table II). While, the average torque present a similar improvement for both winding solutions.
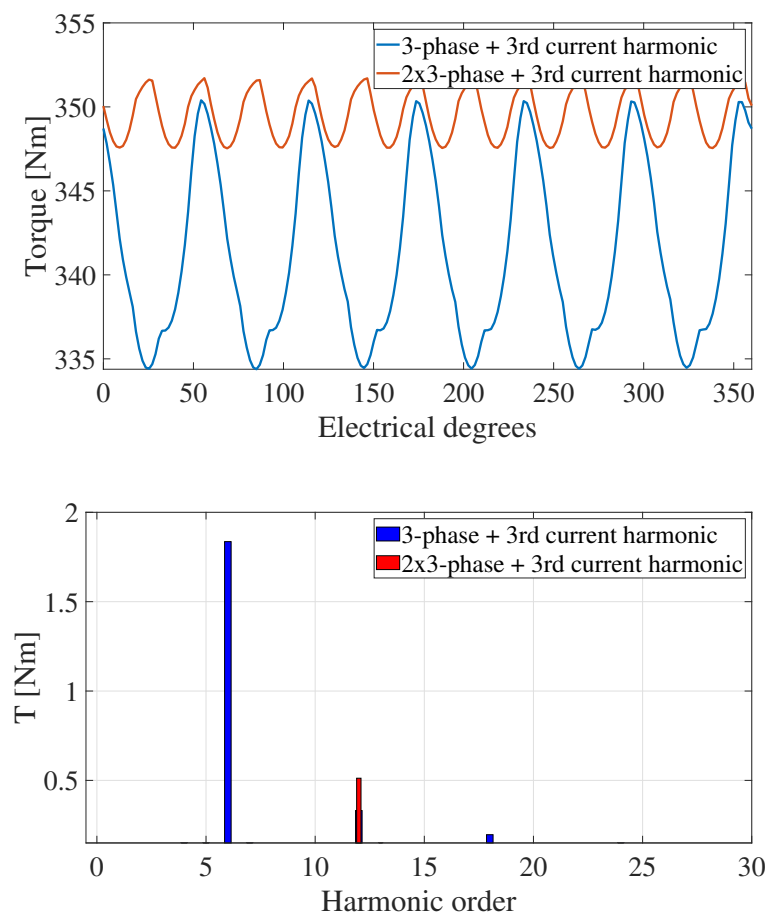

Fig. 9. Torque ripple waveforms for 3-phase and 2x3-phase arrangement with harmonic injection and corresponding FFT.

2) Efficiency: the Juole losses are calculeted considering ideal windings so that the AC component is neglected. The iron losses are slightly higher with respect to the solution without third current injection, whereas the Joule losses are increased significantly due to the fact that more current is fed to the machine (Table IV). However, the efficiency results similar due to the much higher output torque. This can be a good solution when an overload drive capability is required without exceeding the inverter current limit.

\section{CONCLUSion}

This paper analyses the torque performance and efficiency of a dual rotor E-bike machine in a three-phase and dual three-phase winding configurations with and without third harmonic injection. The aim is to identify ways of improving the torque capability. The finite element analyses show that 
TABLE III

TORQUE RIPPLE AND CURRENT

\begin{tabular}{ccc}
\hline Parameters & 3-phase & 2x3-phase \\
\hline Input RMS current [A] & 224.55 & 224.55 \\
Input peak current [A] & 318.18 & 318.18 \\
Average torque [Nm] & 307.85 & 319.05 \\
Torque ripple [\%] & 4.16 & 0.84 \\
\hline & 3-phase + 3rd & 2x3-phase + 3rd \\
\hline Input RMS current [A] & 262.65 & 262.65 \\
Input peak current [A] & 318.18 & 318.18 \\
Average torque [Nm] & 342.4 & 349.6 \\
Torque ripple [\%] & 4.67 & 1.14 \\
\hline
\end{tabular}

TABLE IV

PERFORMANCE COMPARISON BETWEEN 3-PHASE AND 2X3-PHASE ARRANGMENT WITH AND WITHOUT CURRENT HARMONIC INJECTION

\begin{tabular}{ccc}
\hline Parameters & 3-phase & 2x3-phase \\
\hline Iron losses [W] & 1427 & 1420 \\
Joule losses [W] & 3857 & 3857 \\
Average torque [Nm] & 307.85 & 319.05 \\
Efficiency [\%] & 98.21 & 98.60 \\
\hline & 3-phase + 3rd & 2x3-phase + 3rd \\
\hline Iron losses [W] & 1460 & 1476 \\
Joule losses [W] & 5358 & 5358 \\
Average torque [Nm] & 342.4 & 349.6 \\
Efficiency [\%] & 97.94 & 97.97 \\
\hline
\end{tabular}

the multi-phase system gives significant benefits in terms of both average torque and torque ripple. Actually, the average torque improves by $3.64 \%$ whereas the torque ripple decreses by $3.32 \%$. For the proposed dual rotor machine, the third harmonic current injection can increase the average torque. In this way, it is possible to feed the machine with more current without exceeding the inverter current limit. In this operating condition, the output power is improved without affecting the efficiency machine. Actually, the 3-phase system increses the output torque by $11.22 \%$ with third harmonic injection with respect to the solution with only fundamental current component whereas the same comparison for the $2 \times 3$ phase arrangement shows that it presents an improved output torque by $9.57 \%$. Considering the significant torque increment that the third current harmonic injection gives to the machine, it is plausible to imagine that the third current harmonic at constant peak current can be injected only when the Ebike needs more torque whereas it can work without third current harmonic in large part of the time to reduce Joule losses. When the third current harmonic component is injected into the machine, it presents the common mode currents (CMC). This requires the availabilty of the neutral points. The control and electronic hardware for implementing the proposed third harmonic injection technique for high frequency will be subject of future research.

\section{REFERENCES}

[1] L. Parsa, "On advantages of multi-phase machines," in 31st Annual Conference of IEEE Industrial Electronics Society, 2005. IECON 2005. Nov 2005, pp. 6 pp.--

[2] Z. Y. Gu, K. Wang, Z. Q. Zhu, Z. Z. Wu, C. Liu, and R. W. Cao, "Torque improvement in five-phase unequal tooth spm machine by injecting third harmonic current," IEEE Transactions on Vehicular Technology, vol. 67, no. 1, pp. 206-215, Jan 2018.

[3] J. Wang, R. Qu, and L. Zhou, "Dual-rotor multiphase permanent magnet machine with harmonic injection to enhance torque density," IEEE Transactions on Applied Superconductivity, vol. 22, no. 3, pp. 5202204 5202 204, June 2012.

[4] J. Wang, L. Zhou, and R. Qu, "Harmonic current effect on torque density of a multiphase permanent magnet machine," in 2011 International Conference on Electrical Machines and Systems, Aug 2011, pp. 1-6.

[5] Y. Hu, Z. Q. Zhu, and M. Odavic, "Torque capability enhancement of dual three-phase pmsm drive with fifth and seventh current harmonics injection," IEEE Transactions on Industry Applications, vol. 53, no. 5, pp. 4526-4535, Sep. 2017.

[6] M. Slunjski, M. Jones, and E. Levi, "Control of a symmetrical nine-phase pmsm with highly non-sinusoidal back-electromotive force using third harmonic current injection," in IECON 2019 - 45th Annual Conference of the IEEE Industrial Electronics Society, vol. 1, Oct 2019, pp. 969-974.

[7] D. Golovanov, A. Galassini, L. Flanagan, D. Gerada, Z. Xu, and C. Gerada, "Dual-rotor permanent magnet motor for electric superbike," in 2019 IEEE International Electric Machines Drives Conference (IEMDC), May 2019, pp. 951-956.

[8] K. Wang, Z. Q. Zhu, Y. Ren, and G. Ombach, "Torque improvement of dual three-phase permanent-magnet machine with third-harmonic current injection," IEEE Transactions on Industrial Electronics, vol. 62, no. 11, pp. 6833-6844, Nov 2015. 